\title{
The cost of switching between taxonomic and thematic semantics
}

\author{
Jon-Frederick Landrigan ${ }^{1}$ • Daniel Mirman ${ }^{2}$
}

Published online: 12 September 2017

(C) Psychonomic Society, Inc. 2017

\begin{abstract}
Current models and theories of semantic knowledge primarily capture taxonomic relationships (DOG and WOLF) and largely do not address the role of thematic relationships in semantic knowledge (DOG and LEASH). Recent evidence suggests that processing or representation of thematic relationships may be distinct from taxonomic relationships. If taxonomic and thematic relations are distinct, then there should be a cost associated with switching between them even when the task remains constant. This hypothesis was tested using two different semantic-relatedness judgment tasks: Experiment 1 used a triads task and Experiment 2 used an oddball task. In both experiments, participants were faster to respond when the same relationship appeared on consecutive trials than when the relationship types were different, even though the task remained the same and the specific relations were different on each trial. These results are consistent with the theory that taxonomic and thematic relations rely on distinct processes or representations.
\end{abstract}

\section{Keywords Semantic memory $\cdot$ Taxonomic $\cdot$ Thematic}

Semantic knowledge is a crucial aspect of human cognition. It allows us to understand language, interact with objects, remember the past, and imagine future events (Buxbaum \& Saffran, 2002; Irish, Addis, Hodges, \& Piguet, 2012; McRae $\&$ Jones, 2013). The representation, organization, cognitive

Jon-Frederick Landrigan

Jon.Landrigan@gmail.com

1 Department of Psychology, Drexel University, Philadelphia, PA, USA

2 Department of Psychology, University of Alabama at Birmingham, Birmingham, AL, USA algorithms, and neural implementation associated with this knowledge therefore plays an important role in various cognitive processes. Dating back at least to the 19th century, philosophers noted that there were different kinds of relationships within semantic knowledge. One particularly important distinction is between relationships based on similarity and relationships based on contiguity (Bain, 1864). In contemporary cognitive psychology and cognitive neuroscience, concepts are considered similar if they share features (e.g., DOG and WOLF; fur, tail, four legs) and this relationship tends to place concepts into taxonomic structures based on category membership (e.g., animals). On the other hand, contiguity is based on co-occurrence in time and place (e.g., DOG and LEASH; walking a $\operatorname{dog}$ ), which are typically called "thematic" relations. Although research has progressed quickly in regard to the representation and processing of concepts that share similar features, the field has not done as good a job of accounting for the integration and processing of relations based on contiguity and/or thematic semantics.

The taxonomic-thematic distinction is important because these relationships are dissociable and intrinsic to long-term semantic knowledge. Other kinds of semantic relations can be formed on-line in response to task instructions (e.g., ad hoc categories such as "things to save from a burning house" or single-feature categories like "things that are orange"), but these are not part of long-term semantic knowledge and typically engage additional processes such as cognitive control (Lupyan, 2009; Lupyan \& Mirman, 2013). Finally, associative relationships (e.g., those formed in free word association tasks) are defined by the free association task rather than a type of semantic relation and are made up of various types of relationships (e.g., feature based, event based, compound words). For further review of the distinction between taxonomic and thematic semantics from other categories see Mirman, Landrigan, and Britt (2017). 
Contemporary theories of semantic memory agree that semantic knowledge relies on a distributed system that integrates modality-specific representations at various convergence zones and/or levels (Allport, 1985; Binder, Desai, Graves, \& Conant, 2009; Fernandino et al., 2015; Gainotti, 2011; Meyer \& Damasio, 2009). Although a variety of phenomena can be captured without including a "hub" or convergence zones (Cree, McRae, \& McNorgan, 1999; Mirman \& Magnuson, 2008; O’Connor, Cree, \& McRae, 2009; Rabovsky \& McRae, 2014), most models include either one central hub (e.g., (Lambon Ralph, Jefferies, Patterson, \& Rogers, 2017; Rogers, Lambon Ralph, Garrard, et al., 2004) or a series of convergence zones (e.g., McNorgan, Reid, \& McRae, 2011; Meyer \& Damasio, 2009). Studies of the neural basis of semantic cognition have identified at least one central hub in the anterior temporal lobe (ATL; Lambon Ralph et al., 2017; Patterson, Nestor, \& Rogers, 2007). Computational implementations of this huband-spoke model have provided very precise accounts of a broad range of findings from individuals with semantic deficits as well as from neurologically intact participants (Barsalou, Simmons, Barbey, \& Wilson, 2003; Chen, Lambon Ralph, \& Rogers, 2017; Cree \& McRae, 2003; Lambon Ralph et al., 2017; Patterson et al., 2007; Rogers, Lambon Ralph, Garrard, et al., 2004; Rogers, Lambon Ralph, Hodges, \& Patterson, 2004b; Rogers \& Patterson, 2007). However, these models capture phenomena primarily related to taxonomic relationships but ignore the role of thematic relationships. For example, individuals with semantic dementia (also called the semantic variant of primary progressive aphasia) produce a very specific pattern of exclusively taxonomic semantic errors in tasks such as picture naming, word comprehension, and object decision (e.g., Hurley, Paller, Rogalski, \& Mesulam, 2012; Patterson et al., 2007). These error patterns are associated with ATL degeneration and are captured very well by hub-and-spoke computational models.

In contrast, individuals with poststroke aphasia produce both taxonomic (e.g., HORSE for COW) and thematic (e.g., LEASH for DOG) errors in picture naming (Schwartz et al., 2011). In a recent systematic review, we synthesized results from more than 100 studies that examined the distinction between taxonomic and thematic relations (Mirman et al., 2017). Key lines of evidence have shown that taxonomic and thematic relations make independent contributions to semantic relatedness (e.g., Estes, Gibbert, Guest, \& Mazursky, 2012; Wisniewski \& Bassok, 1999), that they have different time courses of activation (e.g., Kalénine, Mirman, Middleton, \& Buxbaum, 2012), and that individuals differ in their reliance on taxonomic versus. thematic relations (e.g., Dunham \& Dunham, 1995; Mirman \& Graziano, 2012), including a neuropsychological double dissociation showing that taxonomic and thematic knowledge can be impaired separately (e.g., Au, Chan, \& Chiu, 2003; Merck, Jonin, Laisney, Vichard, \& Belliard, 2014; Semenza, Bisiacchi, \& Romani, 1992; Semenza, Denes, Lucchese, \& Bisiacchi, 1980).
Jackson, Hoffman, Pobric, and Lambon Ralph (2015) found that taxonomic and thematic processing activated overlapping brain regions ${ }^{1}$ and proposed that a single hub-and-spoke model could account for both kinds of relations by treating thematic relations as a kind of feature. Using their example, the thematic relation between croissants and coffee could be captured by allowing the concept CROISSANT to have a $<$ coffee $>$ feature (or perhaps <eaten with coffee $>$ ). However, on this approach, the <coffee $>$ feature would be completely independent of the COFFEE concept because the hub-and-spoke model makes a strong distinction between concepts, which are representations based on convergent features in the hub, and features, which are represented in the spokes. Therefore, the $<$ coffee $>$ feature that is part of the CROISSANT concept would not be the same as the COFFEE concept, which would have its own set of features. Further, if thematic relations are features, then the CROISSANT$<$ coffee $>$ relationship has nothing in common with other thematic relations, such as BOAT $-<$ river $>$, DOG $-<$ leash $>$. As described below, the present experiments were designed to test this hypothesis - that thematic relations have something in common that is substantively distinct from taxonomic relations.

One possible explanation for the observed differences between taxonomic and thematic relations is that thematic relationships are also based on features, but either the features are different from those that typically form taxonomic relationships, or the features are integrated differently and therefore require a separate processing mechanism (Mirman et al., 2017). This is related to the account proposed by Jackson et al. (2015) but differs in that thematic relations are not themselves features but, like taxonomic relations, are based on convergent, distributed representations over multiple features. The present experiments sought to test this hypothesis by adapting a method that has been previously used to study the role of modality-specific perceptual features in semantic processing (e.g., McNorgan et al., 2011; Pecher, Zeelenberg, \& Barsalou, 2003). In those studies, participants performed a feature verification task (e.g., "BLENDER can be LOUD”). The key finding was that reaction times were about $20 \mathrm{~ms}$ to $40 \mathrm{~ms}$ slower when subsequent trials queried a feature from a different modality (e.g., "CRANBERRIES can be TART") than the same modality (e.g., "LEAVES can be RUSTLING"). This switch cost was interpreted to mean that distinct, modality-specific feature representations were engaged during this task even though the task made no explicit reference to mental imagery or feature modality.

The present experiments used a similar approach but with tasks that tapped multimodal taxonomic or thematic relations.

\footnotetext{
${ }^{1}$ The neural basis of semantic cognition is not the focus of this study, but we note that although Jackson et al. (2015) found no dissociation between taxonomic and thematic neural systems, several other studies have found that they do dissociate, so the existence and nature of a possible neural dissociation remains an open question (for systematic review and discussion, see Mirman et al., 2017).
} 
Following Pecher et al. (2003), participants performed a single semantic-relatedness judgment task throughout each experiment with no explicit instructions about the nature of the taxonomic or thematic relationships. The tasks ensured that the participants would engage their semantic system(s) and that they would identify particular semantic relations without explicit instruction to focus on taxonomic or thematic relatedness. This prevented any potential switch costs from being attributed to higher level explicit task maintenance, updating, or conflict processes. The critical relation on each trial was either taxonomic or thematic, and adjacent trials could have the same kind of relation (e.g., taxonomic to taxonomic or thematic to thematic) or different relations (e.g., taxonomic to thematic or thematic to taxonomic). The taxonomic and thematic relationships used in the present experiments were not reducible to single modalities or feature types. For example, some taxonomic relationships were purely feature based (e.g., SPAGHETTI and WORMS) whereas others were category based (e.g., BOAT and TRAIN). Similarly, the thematic relationships varied as well (e.g., SAW and TREE, MOON and ASTRONAUT). Thus, if a switch cost is observed between taxonomic and thematic trials, it would have to be above and beyond any cost associated with switching between the feature types within a single relationship type, suggesting a commonality among the taxonomic relationships that is distinct from thematic relationships. In sum, if taxonomic and thematic relationships rely on different features or if those features are integrated in different ways, then it should be possible to observe similar switch costs when the subsequent trials differ compared with when they are the same.

\section{Experiment 1: Triads}

\section{Materials and methods}

Participants Thirty-two participants were recruited from Drexel University Psychology courses (24 females). The mean age of the participants was 19.9 years $(S D=1.8)$. All participants were native English speakers or multilingual speakers who had learned English by the age of 5 years. All participants provided informed consent in accordance with procedures approved by the Drexel University Institutional Review Board. Participants received course credit for their participation. No participants were excluded from participation or analysis.

Materials Candidate word pairs were drawn from a prior norming study (Landrigan \& Mirman, 2016). In short, each pair (a reference word and its potential taxonomic and thematic targets) were normed on average by 20 participants in separate surveys using Amazon Mechanical Turk for both their taxonomic relatedness (sharing similar features and/or belonging to the same category; participants rated the word pairs on a scale of $1=$ not similar at all to $7=$ very similar $)$ and their thematic relatedness (co-occurrence in common events; participants rated the word pairs on a scale of $1=$ not related at all to $7=$ very related ). Pairs and/or triads were then considered for inclusion if they showed a clear dichotomy between their taxonomic and thematic relationships. More specifically, the reference and its two targets had to exhibit unique relationships such that the reference and its taxonomic pair word had high taxonomic similarity and low thematic relatedness, whereas the reference and its thematic pair word had high thematic relatedness and low taxonomic similarity. ${ }^{2}$ For the full stimulus list, see Appendix Table 5; for a full description of the norming procedure, see Landrigan and Mirman (2016). Taxonomically and thematically related words were shuffled to become the unrelated items for other triads and normed using the same method as the taxonomic and thematic pairs to control for unforeseen relationships. This shuffling was done to control for other properties of the words that may affect the task; thus, all taxonomic items appeared as both a taxonomically related item for its target word and as the unrelated item for a different target, and the same was done for thematic items. All conditions were also matched on word length in letters and phonemes (obtained from the Speech \& Hearing Lab Neighborhood Database at Washington University in St. Louis), word frequency (Brysbaert \& New, 2009), orthographic neighborhood sizes (Marian, Bartolotti, Chabal, \& Shook, 2012), and imageability obtained from the MRC Psycholinguistic Database (Wilson, 1988); see Table 1.

Four experimental trial lists were created consisting of two blocks each. Lists were counterbalanced so that each reference item appeared in both taxonomic and thematic trials and in "same" (taxonomic-to-taxonomic or thematicto-thematic) and "switch" trials (taxonomic-to-thematic defined as a thematic switch trial or thematic-to-taxonomic defined as a taxonomic switch trial). Each target word appeared once in a block. Within a single list, each reference word was presented in a taxonomic trial in one block and in a thematic trial in the other block; the same/switch counterbalancing was done across lists (and, thus, across participants). In sum, each list contained 96 trials in a 2 (trial type: taxonomic vs. thematic) $\times 2$ (task type: same vs. switch) design, with 24 trials in each cell of the design. Participants were randomly assigned to one of the four total lists, resulting in eight participants completing each list.

\footnotetext{
${ }^{2}$ This dichotomous approach resulted in a bimodal distribution of relationship strengths, which was optimal for the factorial design but precluded a continuous analysis of the association between relationship strength of the word pairs and the observed switch cost. For bimodally distributed data, continuous analyses tend to reproduce categorical analysis results even if the within-group patterns are in the opposite direction (Simpson's paradox), making the continuous analysis redundant and possibly misleading.
} 
Table 1 Mean semantic and lexical properties of all taxonomic and thematic words

\begin{tabular}{llll}
\hline & Taxonomic & Thematic & Comparisons \\
\hline Similarity rating & $4.79(1.01)$ & $2.89(0.87)$ & $t(94)=9.72, p<.001$ \\
Relatedness rating & $3.23(0.94)$ & $5.79(0.87)$ & $t(94)=-13.69, p<.001$ \\
Difference score & $1.55(0.55)$ & $2.90(0.78)$ & $t(94)=-9.72, p<.001$ \\
Number of letters & $5.25(1.98)$ & $5.33(1.65)$ & $t(94)=-0.22, p=0.83$ \\
Number of phonemes & $4.38(1.73)$ & $4.52(1.55)$ & $t(94)=-0.43, p=0.67$ \\
Word frequency $(\log )$ & $1.23(0.6)$ & $1.39(0.51)$ & $t(94)=-1.40, p=0.16$ \\
Orthographic neighborhood & $10.17(10.17)$ & $7.21(7.65)$ & $t(94)=1.59, p=0.11$ \\
Imageability* & $578.47(42.14)$ & $590.48(40.62)$ & $t(67)=-1.20, p=0.23$ \\
\hline
\end{tabular}

Standard errors in parentheses. *Imageability ratings were only available for 69 of the 96 words (72\%)

Procedure The experiment was run using PsychoPy software. Instructions were to select the word that was most related to the word at the top of the screen (i.e., the reference word) by pressing $Z$ for the word on the bottom left and $M$ for the word on the bottom right. The response options were randomly assigned to sides on each trial. Reaction time recordings began at the presentation of the words until the participant's response. Each trial was followed by an intertrial interval (ITI) of $500 \mathrm{~ms}$ before the start of the next trial. After completing five practice trials with feedback, participants were told the experiment would begin and no more feedback would be given. The first five trials after the practice portion were filler trials (not analyzed) to allow for further practice and optimal performance on all analyzed trials. A break was provided midway through the experimental list of trials (between the two blocks). The first trial after the midway break was excluded as it was neither a same nor a switch trial. The trial sequence is illustrated and described in Fig. 1.

\section{Results}

Data were analyzed using multilevel regression implemented in R (Version 3.1.2; R Core Team, 2014). Reaction times of incorrect trials and trials where the reaction time was less than $250 \mathrm{~ms}$ were excluded. The subsequent trial was also excluded because, if the participant responded incorrectly or before processing the stimuli, then it was assumed they did not understand the probed relationship and therefore the subsequent trial would be neither a same nor a switch trial. Exclusion criteria resulted in a total of 218 out of the original 3,072 experimental trials $(7 \%)$ being dropped from further analysis. Trials with the target item WAGON were excluded due to poor overall accuracy $(69 \%)$ across participants $(N=44$ trials). All participants had reasonably high overall accuracy $(M=96 \%$, range: $87 \%-100 \%$ ), so none were excluded from analysis. Error counts by condition are shown in Table 2. After exclusions, the total number of remaining observations was 2,810 . Using the lme4 package (Version 1.1-7), a linear mixed- effects model was employed to analyze the raw reaction time data (Baayen, Davidson, \& Bates, 2008). The model included fixed effects of trial type (taxonomic or thematic) and task type (same or switch), along with random effects of trial type by reference word and trial type and task type by participant (see Table 2 note for model syntax). All $p$ values were estimated using the normal distribution. Table 2 shows modelestimated condition means and standard errors.

Participants were faster to respond to thematic trials $(M=$ $1,771 \mathrm{~ms}, S E=74 \mathrm{~ms})$ than taxonomic trials $(M=2,088 \mathrm{~ms}$, $S E=123 \mathrm{~ms})$, Estimate $=158.55, S E=51.29, p<0.01$. Critically, participants were slower in switch trials $(M=$ $1,965 \mathrm{~ms}, S E=93 \mathrm{~ms})$ than in same trials $(M=1,891 \mathrm{~ms}$, $S E=85 \mathrm{~ms})$, Estimate $=-37.19, S E=17.48, p=0.03$. The interaction between trial type and task type was not statistically significant, Estimate $=-6.96, S E=16.81, p=0.68$.

\section{Discussion}

These results show a clear switch cost: responses were about $74 \mathrm{~ms}$ faster when the kind of relatedness (taxonomic or thematic) was the same as the preceding trial, than when it was the other kind of relatedness. This pattern is consistent with the proposal outlined in the Introduction: taxonomic and thematic relationships may be represented or processed in different ways leading to the observed switch cost. Critically, this switch cost was observed in the absence of any explicit instructions about taxonomic and thematic relationships and across different kinds of taxonomic or thematic relationships.

\section{Experiment 2: Oddball}

To strengthen the evidence from Experiment 1, the switch cost hypothesis was further examined using a more complex semantic judgment task. Prior research suggests that similarity and dissimilarity judgments are not simply the inverse of each other (Golonka \& Estes, 2009; Simmons \& Estes, 2008). To test 


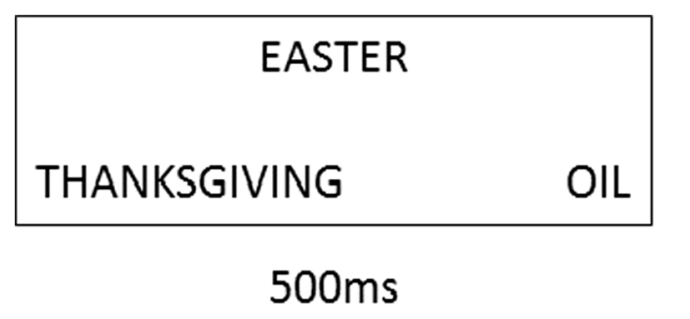

\begin{tabular}{|lll|}
\hline & BOAT & \\
& & \\
FRISBEE & & TRAIN \\
\hline
\end{tabular}

$500 \mathrm{~ms}$

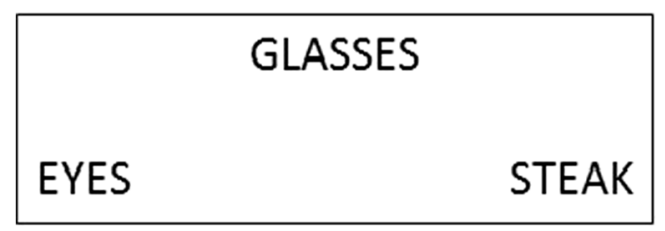

$500 \mathrm{~ms}$

\begin{tabular}{|cc|}
\hline \multicolumn{2}{|r|}{ COSTUME } \\
HALLOWEEN & CORN \\
\hline
\end{tabular}

$500 \mathrm{~ms}$

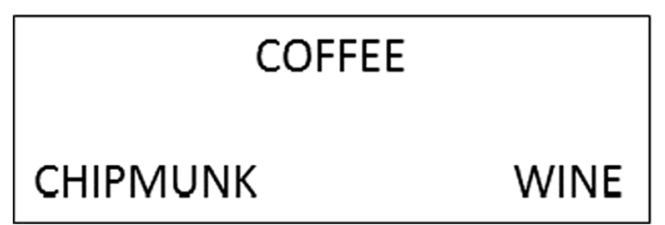

Fig. 1 Example timeline of trials. Each box represents a single trial with a 500-ms ITI between trials. The first (EASTER-THANKSGIVING) and second (BOAT-TRAIN) trials are taxonomic trials and thus the second trial is considered a taxonomic same trial. The third trial (GLASSES-EYES) is a thematic trial and therefore this is considered a thematic switch trial. The fourth trial (COSTUME-HALLOWEEN) in the sequence is also a thematic trial and is therefore a thematic same trial. The final trial (COFFEE-WINE) is once again a taxonomic trial and thus is a taxonomic switch trial

dissimilarity, studies have used an odd-one-out or oddball task in which participants need to process both relatedness and difference in order to identify the oddball, either based on general semantic relatedness or based on specific features (Davidoff \& Roberson, 2004; Lupyan, 2009). Therefore, following this vein, we tested whether the observed switch cost from Experiment 1 would also emerge when participants made semantic difference or oddball judgments. In addition, the oddball task provided an opportunity to test the switch cost when the
Table 2 Experiment 1 results

\begin{tabular}{|c|c|c|c|c|c|}
\hline & \multicolumn{3}{|c|}{ Reaction time (ms): Mean (SE) } & \multicolumn{2}{|c|}{ Error rate } \\
\hline & $\begin{array}{l}\text { Same } \\
\text { trials }\end{array}$ & $\begin{array}{l}\text { Switch } \\
\text { trials }\end{array}$ & $\begin{array}{l}\text { Switch } \\
\text { cost }\end{array}$ & $\begin{array}{l}\text { Same } \\
\text { trials }\end{array}$ & $\begin{array}{l}\text { Switch } \\
\text { trials }\end{array}$ \\
\hline Taxonomic & $2,044(122)$ & $2,132(129)$ & 88 & .04 & .03 \\
\hline Thematic & $1,741(74)$ & $1,801(81)$ & 60 & .03 & .03 \\
\hline
\end{tabular}

Model: ReactionTime $\sim$ TrialType + TaskType + (TaskType $:$ TrialType) $+(1+$ TrialType $\mid$ RefWord $)+(1+$ TrialType + TaskType $\mid$ Participant $)$

two relation types are (hypothetically) in competition with one another. That is, when the critical relation type from one trial needs to be suppressed on the subsequent trial, and when a previously suppressed relation type becomes the critical relation on the next trial.

Experiment 2 used a similar same/switch design as Experiment 1; however, participants had to identify which one of four items was least related to the others. Each trial contained a triplet of items that shared one kind of relationship and an "oddball" item that was related to one of the other items in the opposing relationship. Thus, in order to correctly identify the dominant triplet, participants needed to suppress the opposing relationship. Therefore, when switching between taxonomic and thematic dominance, participants would have to reactivate the previously suppressed relationship in order to correctly identify the new dominant relationship and subsequently identify the oddball. Although this differs from prior oddball experiments (e.g., Lupyan, 2009), using four items allowed us to present participants with both relationships on each trial so that they could compete with one another. This would therefore force participants to engage their semantic systems at an even deeper level than in the triads task as participants need to consider all possible relationships on each trial and suppress and reactivate relationships in order to identify the correct oddballs.

\section{Materials and methods}

Participants Thirty-two participants were recruited from Drexel University Psychology courses (22 females). Recruitment for the two experiments was independent, but two of the overall total of 62 participants completed both experiments. Both of them completed Experiment 2 at least 6 months after completing Experiment 1. The mean age of the participants was 20.8 years $(S D=1.9)$. All participants were native English speakers or multilingual speakers who had learned English by the age of 6 years. All participants provided informed consent in accordance with procedures approved by the Drexel University Institutional Review Board. Participants received course credit for their participation.

Materials Each trial in this experiment consisted of the simultaneous presentation of four words. Three words were related 
either taxonomically or thematically, and the fourth word was related to a reference word in the opposite relationship. Word quartets were constructed by taking the triads from Experiment 1 and adding an extra thematic or taxonomic item. Each quartet was then divided into the four possible threeword subsets, and these triplets were normed using Qualtrics and Mechanical Turk. Participants were asked to rate on a scale of 1 to 7 how well the triplets grouped together based on co-occurrence in time or place or based on similarity (for an example, see Table 3).

Trial quartets were only included in Experiment 2 if the correct triplet had a mean normed rating of at least 0.5 points higher than the other possible triplets for that quartet. That is, any triplet involving the "oddball" had to be at least 0.5 points less well-formed than the correct triplet that omitted the oddball. This relatively low threshold was used in order to maximize the number of possible trials: Excluding error trials was selected over trying to guess which items were likely to elicit many errors. The oddball words and nonoddball words were also matched on word length in letters and phonemes (obtained from the Speech \& Hearing Lab Neighborhood Database at Washington University in St. Louis), word frequency (Brysbaert \& New, 2009), and orthographic neighborhood sizes (Marian et al., 2012). The norming criteria resulted in a final list of 64 quartets (i.e., 32 taxonomic quartets and 32 thematic quartets; see Appendix Table 6 for the full set of stimuli).

Experimental trial lists were created in the same manner as Experiment 1. Note that both kinds of relations were present on each trial: as illustrated in Fig. 2, each trial contained a dominant relation that bound three items (in the example, the thematic relation that binds EASTER, RABBIT, and EGG) and a subordinate relation that bound the oddball with one of the items (in the example, the taxonomic relation between EASTER and THANKSGIVING). In sum, there were 32 trials per block, totaling 64 critical trials per participant in a 2 (trial type: taxonomic vs. thematic) $\times 2$ (task type: same vs. switch) design, with 16 trials in each cell. Participants were randomly assigned to one of the four lists, resulting in eight participants completing each list.

Table 3 Example trial quartet: EGG, RABBIT, EASTER, THANKSGIVING

\begin{tabular}{lll}
\hline Triplet & Oddball & Group rating \\
\hline EASTER, RABBIT, EGG & THANKSGIVING & 6.15 \\
EASTER, RABBIT, THANKSGIVING & EGG & 2.37 \\
EASTER, EGG, THANKSGIVING & RABBIT & 2.35 \\
RABBIT, EGG, THANSGIVING & EASTER & 2.00 \\
\hline
\end{tabular}

Note. Rows show each possible combination of triplet, oddball, and the mean group rating of the triplet. In this example, THANKSGIVING is the correct oddball and all triplets that included it received lower group ratings than the correct triplet that excluded it

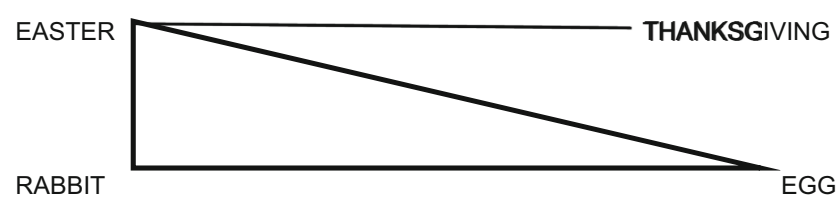

Fig. 2 Example trial presentation with a taxonomic oddball and dominant thematic grouping. Lines indicate relationships and are included in this schematic but not in the actual trials

Procedure The experiment was run using PsychoPy software. Participants were instructed to select the word that does not fit with the others by clicking on the word with the mouse. The words (including the oddball) were randomly assigned to one of four positions/quadrants on each trial. After each trial, the participant was presented with a cross in the middle of the screen, which they needed to click on in order to initiate the next trial (following an ITI of $500 \mathrm{~ms}$ ). This was done so the mouse would be in the center of the screen at the start of each oddball trial. A mouse response was used in place of a key response due to the increased number of response options participants (i.e., two response options in Experiment 1, but four response options in Experiment 2). Thus, in order to eliminate errors from simply hitting the wrong key, the mouse response was used. Figure 3 shows an example trial sequence. Practice trials and breaks were carried out in the same manner as in Experiment 1.

\section{Results}

Accuracy in Experiment 2 was much lower $(M=75 \%)$ than in Experiment 1, most likely due to the more open-ended nature of the task. Each trial contained a dominant relation that bound three items but also a subordinate relation that bound two of the items. Binding a third item into the subordinate relation was all that was required for a participant to make an incorrect response, and given that a lower inclusion threshold was used in order to maximize the number of trials, some of the trials may have fallen victim to alternative interpretations.

Trial exclusion criteria for Experiment 2 were the same as Experiment 1. In total, 845 trials were excluded from analysis out of the original 2,048 experimental trials (41\%). In order to ensure a minimum level of data in each condition, three participants (38 total trials) were excluded from analyses because they did not have at least three analyzable trials in each of the four conditions. The final analyzed data set consisted of 1,165 observations from 29 participants $(M=40$ trials per participant, range: 14-58). Data were analyzed using the same model specifications as Experiment 1 (see Table 2).

Overall, participants were faster to respond to taxonomic trials $(M=5,421 \mathrm{~ms} ; S E=430 \mathrm{~ms})$ than thematic trials $(M=6,526 \mathrm{~ms} ; S E=475 \mathrm{~ms})$, Estimate $=-554.93, S E=$ 221.09, $p=.01$. As in Experiment 1, participants were slower in switch trials $(M=6,227 \mathrm{~ms} ; S E=471 \mathrm{~ms})$ than in same trials $(M=5,688 \mathrm{~ms} ; S E=358 \mathrm{~ms})$, Estimate $=-274.51, S E=$ 

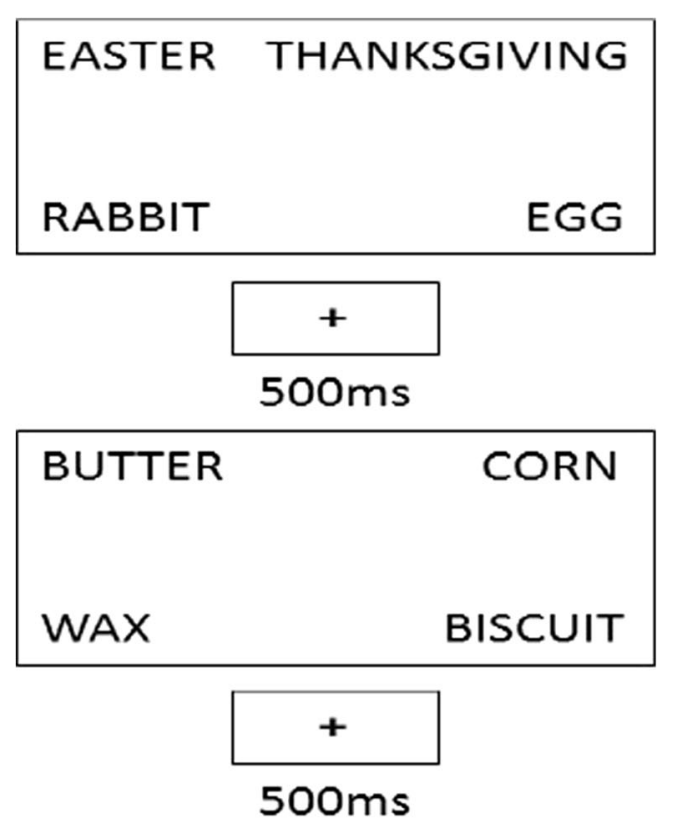

\begin{tabular}{|lr|}
\hline CHIPMUNK & TRAP \\
GERBIL & MOUSE \\
\hline
\end{tabular}

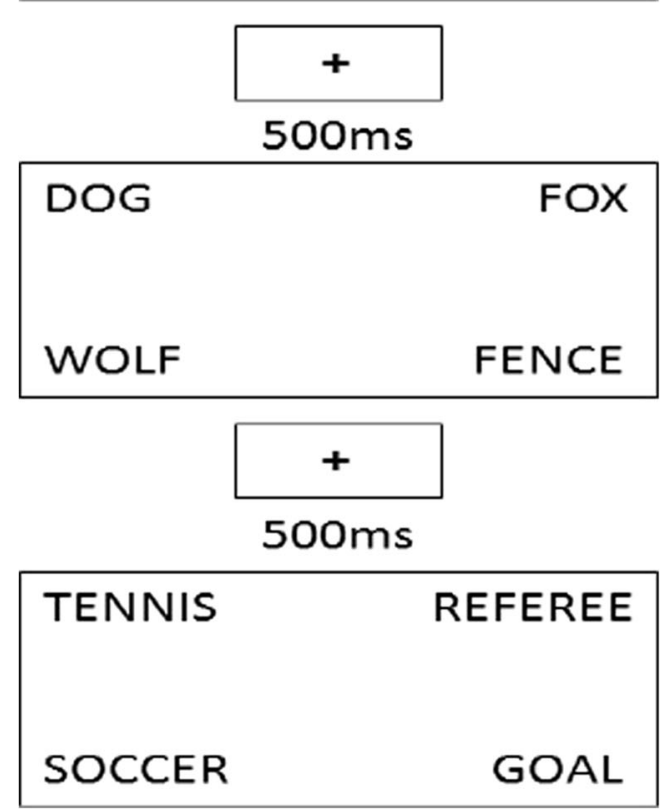

Fig. 3 Example timeline of Experiment 2 trials. Each box represents a single trial with between-trial cross-presentations and 500-ms ITI. The first trial (EASTER-RABBIT-EGG) is a thematic trial and the second trial (BUTTER-CORN-BISCUIT) is a thematic trial as well. Thus, the second trial is a thematic same trial. The third trial (CHIPMUNK-GERBIL-MOUSE) is a taxonomic trial and is therefore a taxonomic switch trial. The fourth trial (DOG-WOLF-FOX) is a taxonomic trial as well and is considered a taxonomic same trial. The last trial in this sequence (SOCCER-GOALREFEREE) is a thematic switch trial
135.99, $p=0.04$. The interaction between trial type and task type was not statistically significant, Estimate $=131.35, S E=$ $99.86, p=.19$ (see Table 4). Table 4 also shows that the errors followed the same pattern as the reaction times: more errors on switch trials than same trials. However, a logistic regression analysis with the same parameters as the reaction time model found no main effect of task type (Estimate $=0.12, S E=0.08$, $p=.10$ ), trial type (Estimate $=0.24, S E=0.18, p=.19)$, nor their interaction (Estimate $=-0.02, S E=0.07, p=.82$ ).

\section{Discussion}

These results converge with the results of Experiment 1: When making an oddball judgment that one item does not fit with the dominant relation (taxonomic or thematic), if the preceding trial relied on the same kind of relation, then responses were about $549 \mathrm{~ms}$ faster than if that preceding trial relied on a different kind of relatedness. This switch cost is again consistent with the proposal that taxonomic and thematic relationships may be processed differently, such that when participants made judgments of the same type on consecutive trials while suppressing the other, their response times were quicker than when they switched between relationships.

\section{General discussion}

The present study set out to test whether taxonomic and thematic relations rely on distinct representations or processing mechanisms. In both experiments, the task instructions were constant for all trials: triads relatedness judgment in Experiment 1, oddball judgment in Experiment 2. However, the materials for each trial were designed to make the participant engage in either taxonomic or thematic processing. The critical finding was that, across both experiments, participants were slower to respond to trials when the dominant relationship in consecutive trials switched (i.e., taxonomic to thematic, or thematic to taxonomic) compared with trials where the dominant relation type remained the same (see Fig. 4). These results suggest that taxonomic and thematic semantic

Table 4 Experiment 2 results

\begin{tabular}{lllllll}
\hline & \multicolumn{2}{l}{ Reaction time $(\mathrm{ms})$ : Mean $(S E)$} & & \multicolumn{2}{l}{ Error rate } \\
\cline { 2 - 4 } \cline { 6 - 7 } & $\begin{array}{l}\text { Same } \\
\text { trials }\end{array}$ & $\begin{array}{l}\text { Switch } \\
\text { trials }\end{array}$ & $\begin{array}{l}\text { Switch } \\
\text { cost }\end{array}$ & & $\begin{array}{l}\text { Same } \\
\text { trials }\end{array}$ & $\begin{array}{l}\text { Switch } \\
\text { trials }\end{array}$ \\
\hline Taxonomic & $5,281(401)$ & $5,567(516)$ & 286 & .18 & .21 \\
Thematic & $6,128(464)$ & $6,940(545)$ & 812 & .21 & .29
\end{tabular}

Model: ReactionTime $\sim$ TrialType + TaskType + (TaskType $:$ TrialType $)$ $+(1+$ TrialType $\mid$ RefWord $)+(1+$ TrialType + TaskType $\mid$ Participant $)$ 


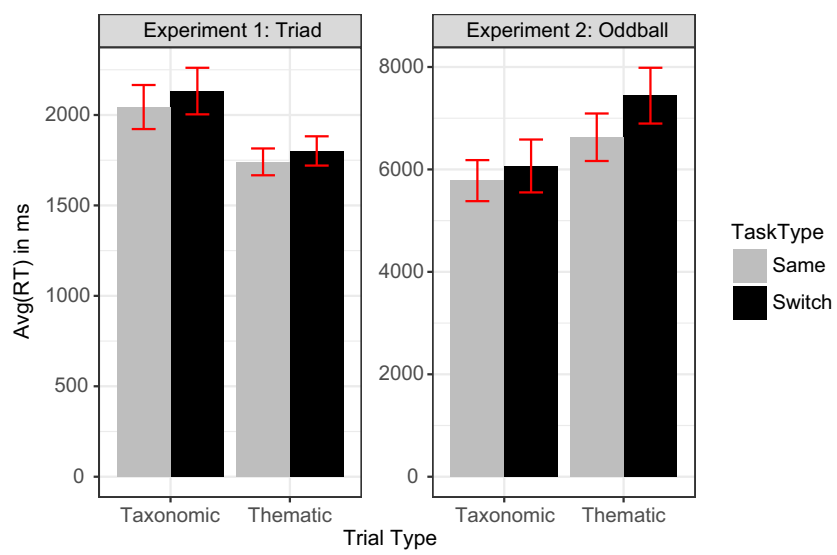

Fig. 4 Results of Experiment 1 (left panel) and Experiment 2 (right panel)

processing are supported by different representations or processing mechanisms.

Within relation type, the individual pairs shared different features and relationship types, so this pattern is not reducible to a modality-specific effect. Any modality-specific effects (such as those demonstrated by Pecher et al., 2003; see also McNorgan et al., 2011) would cause virtually all trials to be "switch" trials, thus washing out the broader taxonomicthematic switch cost. In other words, the variability in the relationship subtypes among thematic relations (e.g., agentpatient, location) and taxonomic relations (e.g., feature based, category based), prevents the observed switch cost effect from being attributed to those lower levels of relation types. At those lower levels, all of the trials would have been switch trials, so there would not have been any additional effect of switching between taxonomic and thematic trials. Similarly, framing thematic relations as one kind of feature in a hub-andspoke system (e.g., Jackson et al., 2015) would not account for the present switch costs because those thematic features (CROISSANT $-<$ coffee $>$, BOAT $-<$ river $>$, DOG-<leash $>$, etc.) would all be distinct, and the cost of switching among them would be just as strong as the cost of switching to a taxonomic relation. Rather, these results indicate that thematic relations, as a group, share some commonality that is distinct from the analogous commonality shared by taxonomic relations. This could be accomplished by gathering all of thematic features into a single spoke and by connecting them to the other spokes so that, for example, the <coffee $>$ feature could be connected to all of the features of the COFFEE concept. But doing so would create a thematic hub - a distinct representational basis for thematic semantics.

There was also a main effect of trial type (i.e., taxonomic vs. thematic) in both experiments. The direction of this effect differed between experiments, most likely due to differences in the task demands. In the oddball experiment, the taxonomic trials may have been easier than thematic trials because the increased number of exemplars made it simpler to identify a common set of features between items or a common category to which the items belonged to (i.e., increased exemplars of a category or sharing features narrowed the search space). In contrast, themes are more flexible in nature, so all items and possible relationships would need to be considered on thematic trials in order to identify the true dominant relationship. A related effect has been observed in developmental studies in which increased numbers of taxonomic exemplars made learning and extending a superordinate label easier for children (Liu, Golinkoff, \& Sak, 2001). In the triads task because there was a defined reference item and participants were only choosing between two response possibilities, a thematic relation may have been easier to detect than a taxonomic relation (a recent free sorting study found that adults exhibited more thematic sorting than taxonomic sorting; Lawson, Chang, \& Wills, 2017). These effects are orthogonal to the observed switch costs that were the main finding of these experiments. The switch cost effects are explicitly consistent with distinct taxonomic and thematic processing mechanisms, and the differential response to task demands only further suggests that these relationships are processed independently of each other.

We argue that distinct taxonomic and thematic representations or processing mechanisms are required to account for the present switch costs. If approached from the opposite view, it is not clear how a single processing mechanism would account for the present results. For example, a standard spreading activation model (e.g. Collins \& Loftus, 1975; McNamara, 1992) does not distinguish between taxonomic and thematic relationships. In such a model, on a given trial, activation would spread to both taxonomically and thematically related concepts. The next trial would involve new, unrelated concepts, and activation would again spread to both taxonomically and thematically related concepts. How long it takes to make a relatedness judgment would depend on strength of connections, but not the type of connection. A basic spreading activation model would need to be extended so that taxonomic and thematic connections differ in some way and use of one connection type facilitates subsequent use of the same type of connections or inhibits use of connections of the other type. This kind of "tagged connections" approach would predict a switch cost, but such a model would be simply one version of distinct taxonomic and thematic semantic systems - in this case, distinct systems of connections. In a hub-and-spoke model, as described above, the thematic features would need to be gathered into a single spoke and connected to the other spokes, which would create a thematic hub that is distinct from the taxonomic hub, in order to account for the switch cost.

Another alternative perspective is to consider relations as dynamic emergent properties of concepts and context. Indeed, context can influence which concept features and relations are activated and how strongly they are activated (e.g., Kalénine et al., 2012; Lee, Middleton, Mirman, Kalénine, \& Buxbaum, 
2013; Yee \& Thompson-Schill, 2016). Although the overall task was constant within each of the present experiments, one could construe each trial context as dynamically emphasizing either taxonomic or thematic relations. However, the actual items and specific relations were different from trial to trial, so this context-specific emphasis would have to operate at the broad level of taxonomic versus thematic relations, independent of the specific items and detailed relations. That is to say, it would also be a way of instantiating the distinct taxonomic and thematic systems.

It is important to acknowledge that semantic cognition does not operate independently of other cognitive systems. In particular, semantic cognition is strongly dependent on cognitive control processes (sometimes called semantic control) that allow manipulation and selection of semantic knowledge to suit task demands (Lambon Ralph et al., 2017; see also Jefferies, 2013) We are not aware of any evidence that either taxonomic or thematic semantic processing requires more semantic control. In fact, the welldocumented neuropsychological double dissociation of taxonomic and thematic semantics argues against differences in the degree of semantic control required for taxonomic and thematic processing (for a review, see Mirman et al., 2017). The reversal of the main effect of task type across our two experiments also argues against any differences in the amount of semantic control required. If differences existed, then the reversal should not have appeared because the relationship requiring more semantic control would have elicited longer reaction times in both experiments. However, it is possible that taxonomic and thematic semantics require different semantic control. A switch cost could then arise as a result of switching between taxonomic control and thematic control processes operating over a single store of semantic knowledge. This interpretation shifts the dissociation from distinct taxonomic and thematic representations to distinct control mechanisms responsible for taxonomic and thematic processing. Further research is necessary to establish whether the dissociation of taxonomic and thematic semantics is in the representation of semantic knowledge or in the processing mechanisms that operate on that knowledge (for a review of the distinction between semantic knowledge and semantic access, see Mirman \& Britt, 2014).

These accounts are not the only possibilities, but they offer demonstrations of why the switch costs reported here require distinct taxonomic and thematic semantic representations or processing mechanisms. The present results do not require that taxonomic and thematic processes are modular - they could be distinct but highly interactive. These results also do not speak to specific neural implementations since the experiments were purely behavioral. From a levels-of-analysis perspective, these results suggest a computational or functional distinction between taxonomic and thematic processing but do not speak to the algorithms or implementations that support this distinction. The degree of separation and interaction between taxonomic and thematic semantic systems, their neural basis, and whether the switch costs observed here are due to facilitation or inhibition are important topics for future research.

Acknowledgements This study was supported by National Institutes of Health Grant R01DC010805 to D.M. and by Drexel University. We thank Allison Britt, Amanda Kraft, and Leah Friedman for help with data collection.

\section{Appendix A}

Table 5 Stimuli used in Experiment 1 (triads)

\begin{tabular}{|c|c|c|c|c|c|c|c|c|}
\hline Reference & Taxonomic & Sim & Rel & Sim - Rel & Thematic & Sim & Rel & Rel - Sim \\
\hline EASTER & THANKSGIVING & 5.70 & 2.61 & 3.10 & RABBIT & 3.31 & 5.98 & 2.67 \\
\hline BOAT & TRAIN & 5.11 & 2.36 & 2.74 & RIVER & 2.90 & 6.04 & 3.14 \\
\hline GLASSES & GOGGLES & 6.11 & 4.78 & 1.33 & EYES & 2.64 & 6.48 & 3.84 \\
\hline HONEY & SAP & 4.96 & 3.33 & 1.64 & BEE & 3.84 & 6.67 & 2.83 \\
\hline SPOON & SHOVEL & 4.57 & 2.56 & 2.01 & TEA & 1.83 & 5.46 & 3.63 \\
\hline BACKРACK & DUFFLE BAG & 6.61 & 4.48 & 2.14 & STUDENT & 2.25 & 6.21 & 3.96 \\
\hline INK & PAINT & 5.86 & 3.72 & 2.14 & PRINTER & 3.64 & 6.29 & 2.65 \\
\hline COSTUME & UNIFORM & 5.90 & 3.48 & 2.42 & HALLOWEEN & 3.50 & 6.46 & 2.96 \\
\hline PEANUT & BEAN & 4.75 & 3.05 & 1.71 & ELEPHANT & 1.65 & 5.09 & 3.44 \\
\hline WAITRESS & CLERK & 4.73 & 2.62 & 2.11 & DINNER & 2.82 & 5.57 & 2.75 \\
\hline SAW & KNIFE & 5.43 & 3.23 & 2.20 & TREE & 1.68 & 4.98 & 3.30 \\
\hline SPAGHETTI & WORMS & 2.61 & 1.27 & 1.35 & TOMATO & 3.57 & 5.88 & 2.31 \\
\hline DORM & APARTMENT & 5.12 & 4.26 & 0.86 & COLLEGE & 3.59 & 6.21 & 2.62 \\
\hline
\end{tabular}


Table 5 (continued)

\begin{tabular}{|c|c|c|c|c|c|c|c|c|}
\hline Reference & Taxonomic & Sim & Rel & Sim - Rel & Thematic & Sim & Rel & Rel - Sim \\
\hline VAULT & BOX & 5.29 & 3.37 & 1.92 & BANK & 4.64 & 6.16 & 1.52 \\
\hline SALT & SUGAR & 5.55 & 3.71 & 1.84 & PRETZEL & 3.32 & 5.74 & 2.42 \\
\hline DISK & FRISBEE & 4.86 & 4.31 & 0.56 & COMPUTER & 3.69 & 6.20 & 2.50 \\
\hline PENCIL & STICK & 4.25 & 2.92 & 1.34 & NOTEBOOK & 3.50 & 6.82 & 3.32 \\
\hline FORK & RAKE & 3.83 & 2.22 & 1.61 & STEAK & 2.43 & 5.48 & 3.05 \\
\hline DOUGH & CLAY & 4.39 & 2.58 & 1.81 & PIZZA & 3.25 & 6.19 & 2.94 \\
\hline SYRUP & OIL & 4.06 & 2.13 & 1.93 & WAFFLE & 3.38 & 6.43 & 3.05 \\
\hline FOOTBALL & BASKETBALL & 5.87 & 3.59 & 2.28 & KICK & 2.61 & 5.86 & 3.25 \\
\hline MOON & BALL & 3.17 & 1.73 & 1.44 & ASTRONAUT & 3.44 & 6.43 & 3.00 \\
\hline OUTLET & KEYHOLE & 3.18 & 2.06 & 1.13 & PLUG & 4.61 & 6.44 & 1.83 \\
\hline PANDA & GRIZZLY & 5.28 & 3.20 & 2.09 & BAMBOO & 2.24 & 4.95 & 2.71 \\
\hline DOG & FOX & 5.04 & 3.37 & 1.67 & FENCE & 1.47 & 4.60 & 3.14 \\
\hline LEASH & ROPE & 5.09 & 3.73 & 1.36 & COLLAR & 4.18 & 6.55 & 2.37 \\
\hline DRAGON & SNAKE & 3.79 & 2.65 & 1.14 & FIRE & 2.14 & 5.79 & 3.65 \\
\hline BUTTER & WAX & 2.97 & 2.23 & 0.74 & CORN & 2.58 & 5.11 & 2.54 \\
\hline SQUIRREL & RAT & 5.23 & 3.25 & 1.98 & NUT & 2.25 & 6.32 & 4.07 \\
\hline RING & WHEEL & 3.69 & 2.29 & 1.40 & DIAMOND & 4.01 & 6.14 & 2.13 \\
\hline CROWN & HAT & 5.29 & 4.15 & 1.14 & QUEEN & 3.08 & 5.87 & 2.79 \\
\hline CHURCH & STORE & 2.61 & 1.48 & 1.13 & BELL & 2.00 & 5.78 & 3.78 \\
\hline BRICK & BOULDER & 3.71 & 2.86 & 0.85 & WALL & 3.85 & 5.79 & 1.95 \\
\hline DUCK & SWAN & 5.99 & 4.88 & 1.11 & TUB & 1.53 & 3.23 & 1.70 \\
\hline WAGON & CAR & 4.54 & 3.48 & 1.06 & FRUIT & 1.07 & 2.53 & 1.46 \\
\hline OCEAN & LAKE & 5.59 & 4.12 & 1.48 & SHARK & 2.44 & 6.17 & 3.73 \\
\hline GUN & SWORD & 4.79 & 3.34 & 1.45 & $\mathrm{COP}$ & 2.25 & 6.36 & 4.11 \\
\hline SIGN & LABEL & 5.70 & 4.35 & 1.35 & STREET & 2.75 & 5.66 & 2.91 \\
\hline NEEDLE & PIN & 5.99 & 5.17 & 0.82 & DOCTOR & 1.81 & 5.28 & 3.48 \\
\hline STEWARDESS & NURSE & 4.29 & 2.16 & 2.13 & AIRPLANE & 2.36 & 6.48 & 4.13 \\
\hline MOUSE & CHIPMUNK & 5.08 & 3.16 & 1.92 & HOLE & 1.63 & 5.18 & 3.55 \\
\hline BREAKFAST & LUNCH & 5.39 & 4.07 & 1.32 & BACON & 4.53 & 6.28 & 1.75 \\
\hline TURNIP & BEET & 5.66 & 4.37 & 1.30 & SALAD & 3.40 & 4.06 & 0.67 \\
\hline OYSTER & SCALLOP & 5.59 & 4.65 & 0.94 & PEARL & 3.75 & 6.50 & 2.75 \\
\hline EDITOR & HOST & 2.32 & 1.59 & 0.74 & ARTICLE & 3.04 & 6.69 & 3.65 \\
\hline ANCHOR & WEIGHT & 5.64 & 3.90 & 1.74 & NAVY & 2.50 & 4.61 & 2.11 \\
\hline SOCCER & TENNIS & 4.32 & 3.55 & 0.77 & GOAL & 2.79 & 6.65 & 3.86 \\
\hline COFFEE & WINE & 4.27 & 2.77 & 1.50 & MUG & 3.06 & 6.54 & 3.49 \\
\hline
\end{tabular}

\section{Appendix B}

Table 6 Stimuli used in Experiment 2 (oddball)

\begin{tabular}{llll}
\hline Reference & Related Item 1 & Related Item 2 & Oddball \\
\hline Taxonomic trials & & & SHIP \\
ANCHOR & WEIGHT & DUMBBELL & STUDENT \\
BACKPACK & SUITCASE & PURSE & WAVE \\
BOAT & TRAIN & BUS & EGG \\
BREAKFAST & LUNCH & SNACK & ROOF \\
BRICK & BOULDER & CEMENT & CORN
\end{tabular}


Table 6 (continued)

\begin{tabular}{|c|c|c|c|}
\hline Reference & Related Item 1 & Related Item 2 & Oddball \\
\hline $\mathrm{CHURCH}$ & COURT & STORE & CROSS \\
\hline COFFEE & WINE & SODA & DONUT \\
\hline COSTUME & UNIFORM & DISGUISE & PLAY \\
\hline CROWN & HAT & YARMULKE & CASTLE \\
\hline DISK & FRISBEE & SAUCER & COMPUTER \\
\hline DOG & FOX & WOLF & FENCE \\
\hline DORM & APARTMENT & BARRACK & FRESHMAN \\
\hline DOUGH & CLAY & MORTAR & PIZZA \\
\hline DRAGON & SNAKE & IGUANA & FIRE \\
\hline EASTER & THANKSGIVING & CHRISTMAS & RABBIT \\
\hline FOOTBALL & BASKETBALL & HOCKEY & TACKLE \\
\hline FORK & RAKE & $\mathrm{HOE}$ & STEAK \\
\hline HEART & STOMACH & KIDNEY & VALENTINE \\
\hline HONEY & JELLY & KETCHUP & $\mathrm{BEE}$ \\
\hline MOUSE & CHIPMUNK & GERBIL & TRAP \\
\hline NEEDLE & THORN & SPIKE & THREAD \\
\hline OUTLET & KEYHOLE & SLOT & ELECTRIC \\
\hline PANDA & GRIZZLY & LION & BAMBOO \\
\hline PEANUT & BEAN & WALNUT & ELEPHANT \\
\hline SALT & SUGAR & SAND & FRIES \\
\hline SAW & KNIFE & AXE & TREE \\
\hline SIGN & LABEL & BILLBOARD & DIRECTIONS \\
\hline SOCCER & TENNIS & LACROSSE & GOAL \\
\hline SQUIRREL & RAT & HAMSTER & NUT \\
\hline STEWARDESS & NURSE & TECHNICIAN & AIRPLANE \\
\hline VAULT & BOX & CONTAINER & MONEY \\
\hline \multicolumn{4}{|l|}{ Thematic trials } \\
\hline ANCHOR & NAVY & SHIP & DUMBBELL \\
\hline BACKPACK & BOOKS & STUDENT & PURSE \\
\hline BOAT & RIVER & WAVE & BUS \\
\hline BREAKFAST & BACON & EGG & SNACK \\
\hline BRICK & ROOF & WALL & BOULDER \\
\hline BUTTER & CORN & BISCUIT & WAX \\
\hline $\mathrm{CHURCH}$ & BELL & CROSS & COURT \\
\hline COFFEE & DONUT & CREAM & SODA \\
\hline COSTUME & HALLOWEEN & PLAY & UNIFORM \\
\hline CROWN & QUEEN & CASTLE & YARMULKE \\
\hline DISK & MUSIC & COMPUTER & FRISBEE \\
\hline DOG & FENCE & HOUSE & WOLF \\
\hline DORM & COLLEGE & FRESHMAN & BARRACK \\
\hline DOUGH & PIZZA & NOODLE & CLAY \\
\hline DRAGON & FIRE & KNIGHT & IGUANA \\
\hline EASTER & RABBIT & EGG & THANKSGIVING \\
\hline FOOTBALL & KICK & TACKLE & BASKETBALL \\
\hline FORK & FONDUE & STEAK & RAKE \\
\hline HEART & VALENTINE & LOVE & STOMACH \\
\hline HONEY & BEE & BEAR & JELLY \\
\hline MOUSE & CHEESE & TRAP & GERBIL \\
\hline NEEDLE & THREAD & SEAMSTRESS & SPIKE \\
\hline
\end{tabular}


Table 6 (continued)

\begin{tabular}{llll}
\hline Reference & Related Item 1 & Related Item 2 & Oddball \\
\hline OUTLET & ELECTRIC & PLUG & KEYHOLE \\
PANDA & BAMBOO & FOREST & LION \\
PEANUT & ELEPHANT & ZOO & BEAN \\
SALT & FRIES & PRETZEL & SAND \\
SAW & TREE & CARPENTER & KNIFE \\
SIGN & STREET & DIRECTIONS & LABEL \\
SOCCER & GOAL & REFEREE & TENNIS \\
SQUIRREL & NUT & NEST & RAT \\
STEWARDESS & AIRPLANE & BEVERAGE & NURSE \\
VAULT & BANK & MONEY & CONTAINER \\
\hline
\end{tabular}

\section{References}

Allport, D. A. (1985). Distributed memory, modular subsystems and dysphasia. In S.K. Newman \& R. Epstein (Eds.), Current perspectives in dysphasia (pp. 32-60). Edinburgh, UK: Churchill Livingstone.

Au, A., Chan, A. S., \& Chiu, H. (2003). Conceptual organization in Alzheimer's dementia. Journal of Clinical and Experimental Neuropsychology, 25(6), 737-750. doi:https://doi.org/10.1076/ jcen.25.6.737.16468

Baayen, R. H., Davidson, D. J., \& Bates, D. M. (2008). Mixed-effects modeling with crossed random effects for subjects and items. Journal of Memory and Language, 59(4), 390-412. doi:https://doi. org/10.1016/j.jml.2007.12.005

Bain, A. (1864). The senses and the intellect. London: Longman, Roberts, \& Green.

Barsalou, L. W., Simmons, W. K., Barbey, A. K., \& Wilson, C. D. (2003). Grounding conceptual knowledge in modality-specific systems. Trends in Cognitive Sciences, 7(2), 84-91.

Binder, J. R., Desai, R. H., Graves, W. W., \& Conant, L. L. (2009). Where is the semantic system? A critical review and meta-analysis of 120 functional neuroimaging studies. Cerebral Cortex, 19(December), 2767-2796. doi:https://doi.org/10.1093/cercor/bhp055

Brysbaert, M., \& New, B. (2009). Moving beyond Kucera and Francis: A critical evaluation of current word frequency norms and the introduction of a new and improved word frequency measure for American English. Behavior Research Methods, 41(4), 977-990.

Buxbaum, L. J., \& Saffran, E. M. (2002). Knowledge of object manipulation and object function: Dissociations in apraxic and nonapraxic subjects. Brain and Language, 82(2), 179-199. doi:https://doi.org/ 10.1016/S0093-934X(02)00014-7

Chen, L., Lambon Ralph, M. A., \& Rogers, T. T. (2017). A unified model of human semantic knowledge and its disorders. Nature Human Behaviour, 1(March), 39. doi:https://doi.org/10.1038/s41562-016-0039

Cree, G. S., \& McRae, K. (2003). Analyzing the factors underlying the structure and computation of the meaning of chipmunk, cherry, chisel, cheese, and cello (and many other such concrete nouns). Journal of Experimental Psychology., 132(2), 163-201. doi:https:// doi.org/10.1037/0096-3445.132.2.163

Cree, G. S., McRae, K., \& McNorgan, C. (1999). An attractor model of lexical conceptual processing: Simulating semantic priming. Cognitive Science, 23(3), 371-414. doi:https://doi.org/10.1016/ S0364-0213(99)00005-1

Davidoff, J. B., \& Roberson, D. (2004). Preserved thematic and impaired taxonomic categorisation: A case study. Language and Cognitive
Processes, 19(1), 137-174. doi:https://doi.org/10.1080/ 01690960344000125

Dunham, P. J., \& Dunham, F. (1995). Developmental antecedents of taxonomic and thematic strategies at 3 years of age. Developmental Psychology, 31(3), 483-493.

Estes, Z., Gibbert, M., Guest, D., \& Mazursky, D. (2012). A dual-process model of brand extension: Taxonomic feature-based and thematic relation-based similarity independently drive brand extension evaluation. Journal of Consumer Psychology, 22(1), 86-101. doi:https:// doi.org/10.1016/j.jcps.2011.11.002

Fernandino, L., Humphries, C. J., Seidenberg, M. S., Gross, W. L., Conant, L. L., \& Binder, J. R. (2015). Predicting brain activation patterns associated with individual lexical concepts based on five sensory-motor attributes. Neuropsychologia, 76, 17-26. doi:https:// doi.org/10.1016/j.neuropsychologia.2015.04.009

Gainotti, G. (2011). The organization and dissolution of semanticconceptual knowledge: Is the "amodal hub" the only plausible model? Brain and Cognition, 75(3), 299-309. doi:https://doi.org/10. 1016/j.bandc.2010.12.001

Golonka, S., \& Estes, Z. (2009). Thematic relations affect similarity via commonalities. Journal of Experimental Psychology. Learning, Memory, and Cognition, 35(6), 1454-1464. doi:https://doi.org/10. 1037/a0017397

Hurley, R. S., Paller, K. A., Rogalski, E. J., \& Mesulam, M. M. (2012). Neural mechanisms of object naming and word comprehension in primary progressive aphasia. Journal of Neuroscience, 32(14), 4848-4855. doi:https://doi.org/10.1523/JNEUROSCI.5984-11.2012

Irish, M., Addis, D. R., Hodges, J. R., \& Piguet, O. (2012). Considering the role of semantic memory in episodic future thinking: Evidence from semantic dementia. Brain, 135(7), 2178-2191. doi:https://doi. org/10.1093/brain/aws119

Jackson, R. L., Hoffman, P., Pobric, G., \& Lambon Ralph, M. A. (2015). The neural correlates of semantic association versus conceptual similarity. Cerbral Cortex, 20(2010), 8524. doi:https://doi.org/10.1093/ cercor/bhv003

Jefferies, E. (2013). The neural basis of semantic cognition: Converging evidence from neuropsychology, neuroimaging and TMS. Cortex, 49(3), 611-625. doi:https://doi.org/10.1016/j.cortex.2012.10.008

Kalénine, S., Mirman, D., Middleton, E. L., \& Buxbaum, L. J. (2012). Temporal dynamics of activation of thematic and functional knowledge during conceptual processing of manipulable artifacts. Journal of Experimental Psychology: Learning, Memory, and Cognition, 38(5), 1274-1295. doi:https://doi.org/10.1037/a0027626

Lambon Ralph, M. A., Jefferies, E., Patterson, K., \& Rogers, T. T. (2017). The neural and computational bases of semantic cognition. Nature 
Reviews Neuroscience, 18(1), 42-55. doi:https://doi.org/10.1038/ nrn.2016.150

Landrigan, J.-F., \& Mirman, D. (2016). Taxonomic and Thematic Relatedness Ratings for 659 Word Pairs. Journal of Open Pscychology Data, 4, 1-4.

Lawson, R., Chang, F., \& Wills, A. J. (2017). Free classification of large sets of everyday objects is more thematic than taxonomic. Acta Psychologica, 172, 26-40. doi:https://doi.org/10.1016/j.actpsy. 2016.11.001

Lee, C., Middleton, E. L., Mirman, D., Kalénine, S., \& Buxbaum, L. J. (2013). Incidental and context-responsive activation of structure- and function-based action features during object identification. Journal of Experimental Psychology Human Perceptual Performance, 39(1), 257-270. doi:https://doi.org/10.1037/a0027533

Liu, J., Golinkoff, R. M., \& Sak, K. (2001). One cow does not an animal make: Young children can extend novel words at the superordinate level. Child Development, 72(6), 1674-94. doi:https://doi.org/10. 1111/1467-8624.00372

Lupyan, G. (2009). Extracommunicative functions of language: Verbal interference causes selective categorization impairments. Psychonomic Bulletin \& Review, 16(4), 711-718. doi:https://doi. org/10.3758/PBR.16.5.986

Lupyan, G., \& Mirman, D. (2013). Linking language and categorization: Evidence from aphasia. Cortex, 49(5), 1187-1194. doi:https://doi. org/10.1016/j.cortex.2012.06.006

Marian, V., Bartolotti, J., Chabal, S., \& Shook, A. (2012). CLEARPOND: Cross-linguistic easy-access resource for phonological and orthographic neighborhood densities. PLOS ONE, 7(8).

McNorgan, C., Reid, J., \& McRae, K. (2011). Integrating conceptual knowledge within and across representational modalities. Cognition, 118, 211-233. doi:https://doi.org/10.1016/j.cognition.2010.10.017

McRae, K., \& Jones, M. (2013). Semantic memory. In D. Reisberg (Ed.), The Oxford handbook of cognitive psychology (pp. 206-219). Retrieved from http://books.google.co.il/books?id= ktFMAgAAQBAJ

Merck, C., Jonin, P. Y., Laisney, M., Vichard, H., \& Belliard, S. (2014). When the zebra loses its stripes but is still in the savannah: Results from a semantic priming paradigm in semantic dementia. Neuropsychologia, 53(1), 221-232. doi:https://doi.org/10.1016/j. neuropsychologia.2013.11.024

Meyer, K., \& Damasio, A. (2009). Convergence and divergence in a neural architecture for recognition and memory. Trends in Neurosciences, 32(7), 376-382. doi:https://doi.org/10.1016/j.tins.2009.04.002

Mirman, D., \& Britt, A. E. (2014). What we talk about when we talk about access deficits. Philosophical Transactions of the Royal Society B: Biological Sciences, 369(1634), 1-14. doi:https://doi. org/10.1098/rstb.2012.0388

Mirman, D., \& Graziano, K. M. (2012). Individual differences in the strength of taxonomic versus thematic relations. Journal of Experimental Psychology: General, 141(4), 601-609. doi:https:// doi.org/10.1037/a0026451

Mirman, D., Landrigan, J.-F., \& Britt, A. E. (2017). Taxonomic and thematic semantic systems. Psychological Bulletin, 143(5), 499-520. doi:https://doi.org/10.1037/bul0000092

Mirman, D., \& Magnuson, J. S. (2008). Attractor dynamics and semantic neighborhood density: Processing is slowed by near neighbors and speeded by distant neighbors. Journal of Experimental Psychology: Learning, Memory, and Cognition, 34(1), 65-79. doi:https://doi.org/ 10.1037/0278-7393.34.1.65
O’Connor, C. M., Cree, G. S., \& McRae, K. (2009). Conceptual hierarchies in a flat attractor network: Dynamics of learning and computations. Cognitive Science, 33(4), 665-708. doi:https://doi.org/10. $1111 / \mathrm{j} .1551-6709.2009 .01024 . \mathrm{x}$

Patterson, K., Nestor, P. J., \& Rogers, T. T. (2007). Where do you know what you know? The representation of semantic knowledge in the human brain. Nature Reviews Neuroscience, 8(December), 976-987. doi:https://doi.org/10.1038/nrn2277

Pecher, D., Zeelenberg, R., \& Barsalou, L. W. (2003). Verifying different madality properties for concepts produces swtiching costs. Psychological Science, 14(2), 119-124.

Rabovsky, M., \& McRae, K. (2014). Simulating the N400 ERP component as semantic network error: Insights from a featurebased connectionist attractor model of word meaning. Cognition, 132(1), 68-89. doi:https://doi.org/10.1016/j.cognition.2014.03.010

R Core Team (2014). R: A language and environment for statistical computing. R Foundation for Statistical Computing, Vienna, Austria. https://www.R-project.org/.

Rogers, T. T., Lambon Ralph, M. A, Garrard, P., Bozeat, S., McClelland, J. L., Hodges, J. R., \& Patterson, K. (2004a). Structure and deterioration of semantic memory: A neuropsychological and computational investigation. Psychological Review, 111(1), 205-235. doi:https:// doi.org/10.1037/0033-295X.111.1.205

Rogers, T. T., Lambon Ralph, M. A., Hodges, J. R., \& Patterson, K. (2004b). Natrual selection: The impact of semantic impairment on lexical and object decision. Cognitive Neuropsychology, 21, 331-352. doi:https://doi.org/10.1016/j.limno.2013.04.005

Rogers, T. T., \& Patterson, K. (2007). Object categorization: Reversals and explanations of the basic-level advantage. Journal of Experimental Psychology: General, 136(3), 451-469. doi:https:// doi.org/10.1037/0096-3445.136.3.451

Schwartz, M. F., Kimberg, D. Y., Walker, G. M., Brecher, A., Faseyitan, O. K., Dell, G. S., . . . Coslett, H. B. (2011). Neuroanatomical dissociation for taxonomic and thematic knowledge in the human brain. Proceedings of the National Academy of Sciences of the United States of America 108, 8520-8524. doi:https://doi.org/10. 1073/pnas.1014935108

Semenza, C., Bisiacchi, P. S., \& Romani, L. (1992). Naming disorders and semantic representations. Journal of Psycholinguistic Research, 21(5), 349-364. doi:https://doi.org/10.1007/BF01067920

Semenza, C., Denes, G., Lucchese, D., \& Bisiacchi, P. (1980). Selective deficit of conceptual structures in aphasia: Class versus thematic relations. Brain and Language, 10(2), 243-248. doi:https://doi.org/ 10.1016/0093-934X(80)90054-1

Simmons, S., \& Estes, Z. (2008). Individual differences in the perception of similarity and difference. Cognition, 108(3), 781-795. doi:https:// doi.org/10.1016/j.cognition.2008.07.003

Wilson, M. D. (1988). The MRC Psycholinguistic Database: Machine Readable Dictionary (Version 2). Behavior ResearchMethods, Instruments, \& Computers, 20, 6-11.

Wisniewski, E. J., \& Bassok, M. (1999). What makes a man similar to a tie? Stimulus compatibility with comparison and integration. Cognitive Psychology, 39, 208-238. doi:https://doi.org/10.1006/ cogp.1999.0723

Yee, E., \& Thompson-Schill, S. L. (2016). Putting concepts into context. Psychonomic Bulletin \& Review, 23(4), 1015-1027. doi:https://doi. org/10.3758/s13423-015-0948-7 